\title{
Phase-contrast Enhanced Mammography: A New Diagnostic Tool for Breast Imaging
}

\author{
Zhentian Wang $^{\mathrm{a}}$, Thomas Thuering ${ }^{\mathrm{a}}$, Christian David $^{\mathrm{b}}$, Ewald Roessl ${ }^{\mathrm{c}}$, \\ Mafalda Trippel $^{\mathrm{d}}$, Rahel A. Kubik-Huch ${ }^{\mathrm{e}}$, Gad Singer ${ }^{\mathrm{d}}$, Michael K. Hohl ${ }^{\mathrm{f}}$, \\ Nik Hauser ${ }^{\mathrm{f}}$ and Marco Stampanoni ${ }^{\mathrm{a}, \mathrm{g}}$ \\ ${ }^{a}$ Swiss Light Source, Paul Scherrer Institut, 5232 Villigen, Switzerland \\ ${ }^{b}$ Laboratory for Micro and Nanotechnology, Paul Scherrer Institut, 5232 Villigen, Switzerland \\ ${ }^{c}$ Philips Technologie GmbH, Röntgenstrasse 24, 22335 Hamburg, Germany \\ ${ }^{d}$ Institute of Pathology, Kantonsspital Baden, 5404 Baden, Switzerland \\ ${ }^{e}$ Department of Radiology, Kantonsspital Baden, 5404 Baden, Switzerland \\ ${ }^{f}$ Department of Gynecology and Obstetrics, Interdisciplinary Breast Center Baden, Kantonsspital Baden, \\ 5404 Baden, Switzerland \\ ${ }^{g}$ Institute for Biomedical Engineering, University and ETH Zürich, 8092 Zürich, Switzerland
}

\begin{abstract}
Phase contrast and scattering-based X-ray imaging can potentially revolutionize the radiological approach to breast imaging by providing additional and complementary information to conventional, absorption-based methods. We investigated native, non-fixed whole breast samples using a grating interferometer with an X-ray tube-based configuration. Our approach simultaneously recorded absorption, differential phase contrast and small-angle scattering signals. The results show that this novel technique - combined with a dedicated image fusion algorithm - has the potential to deliver enhanced breast imaging with complementary information for an improved diagnostic process.
\end{abstract}

Keywords: Differential phase contrast imaging, mammography, grating interferometry, microcalcification

PACS: $41.50 .+\mathrm{h}, 42.30 . \mathrm{Va}, 42.30 . \mathrm{Rx}$

\section{INTRODUCTION}

Breast cancer is the most common cancer among women and the second leading cause of cancer deaths in the world. Current absorption-based mammography bears some limitations, such as low contrast between benign and malignant tissue [1]. During the past few years, phase-contrast and scattering-based X-ray imaging have shown their potential for revolutionizing the radiological approach to breast imaging. We investigated nonfixed, whole-breast samples using a grating interferometer operated with a conventional $\mathrm{X}$-ray source. This novel approach can simultaneously record differential phase and small-angle scattering signals of the specimen, as well as the conventional absorption signal. Our first results demonstrate that the complementary information obtained can improve the diagnostic process for breast cancer, for example to answer clinically relevant, unresolved questions about cancer-invaded regions within healthy tissue.

International Workshop on X-ray and Neutron Phase Imaging with Gratings

AIP Conf. Proc. 1466, 103-106 (2012); doi: 10.1063/1.4742276

(C) 2012 American Institute of Physics 978-0-7354-1072-5/\$30.00 


\section{METHOD}

\section{Talbot-Lau Grating Interferometer}

The principle of grating interferometer for phase contrast imaging has been well developed in the last ten years [2-4]. A Talbot-Lau grating interferometer for mammography (named MammoDPC) has been set up at Paul Scherrer Institut in Villigen (Switzerland). The system consists of a Seifert ID $3000 \mathrm{X}$-ray generator and an unfiltered tungsten line focus tube which is operated at $40 \mathrm{kVp}$ with a mean energy of $28 \mathrm{keV}$ and a current of $25 \mathrm{~mA}$. It uses a three gratings interferometer (with periods of $\mathrm{p}_{0}=14 \mu \mathrm{m}, \mathrm{p}_{1}=$ $3.5 \mu \mathrm{m}$ and $\mathrm{p}_{2}=2.0 \mu \mathrm{m}$ ) and a Hamamatsu C9732DK flat panel CMOS detector featuring a $12 \times 12 \mathrm{~cm}$ field of view with $50 \times 50$ microns pixel size. The distance from the source to the G1 grating was $140 \mathrm{~cm}$. The resulting field of view, limited by the size of the G1 and G2 gratings, was $6 \times 6 \mathrm{~cm}^{2}$. For imaging larger breast tissue samples, multiple acquisitions have been acquired and stitched together.

\section{Sample Preparation and Imaging Protocol}

For the first time ever, we managed to obtain native, non-fixed whole breast samples in collaboration with the Kantonsspital Baden, Switzerland. These samples are measured with an imaging protocol as similar as possible to in-vivo situations, to explore the clinical potential of MammoDPC as far as possible. Native breast tissue was obtained directly after mastectomy and mounted inside a dedicated, cooled breast-tissue holder designed to provide adequate compression of the tissue compared with the in-vivo situation. Ex-vivo mammograms and mammoDPC imaging were then performed at the hospital and at the Paul Scherrer Institute, respectively. Standard histopathological examination followed the MammoDPC acquisition. All results, including MRI, ultrasound, in-vivo and ex-vivo mammograms, and MammoDPC, were then discussed in regularly-scheduled interdisciplinary meetings, which included radiologists, pathologists, breast surgeons, and physicists.

\section{RESULTS}

\section{Enhanced Breast Imaging}

As essentially distinct from conventional mammography, a rising question for MammoDPC is how to effectively fuse absorption, differential phase contrast and scattering signals into one single, but more informative image. This image fusion problem is crucial in order to enable radiologists to adopt the MammoDPC technique in clinical applications. Herein we proposed an effective fusion scheme based on Principal Component Analysis (PCA). The fundamental idea of this scheme contains two steps: first fuse the absorption and scattering images by PCA; then merge the differential phase image to the PCA-fused image to achieve edge enhancement. The resulting image is a grey-level image with similar appearance to conventional radiography images but with an 
edge enhancement effect and enhanced details. The redundant information in the absorption and scattering images is reduced by PCA and the edges are enhanced by the differential phase contrast image. It is worth mentioning that the fusion image can also be post-processed by conventional image processing algorithm to achieve better contrast enhancement.

An interesting example is given in Fig. 1, where we investigated a fresh breast sample with a large tumor mass and many spiculations. The fusion image clearly enhances the spiculations features. This enhancement is diagnostically very relevant since spiculations are usually appearing in relation to malignant formations sometimes difficult to detect in normal, absorption based mammogram. Further, fat tissue usually gives less scattering than glandular tissue and is not helpful to diagnosis in most cases. By PCA fusion, signals from the fat tissue are significantly reduced, as shown in Fig. 1 (b) that the 'cloudy' background is much less in the fusion image than in the absorption image. The ROIs shown in Fig. 1 (c) and (d) highlight the edge enhancement effect contributed by the differential phase signals. The spiculations extending from the malignant tissue are much more clearly seen in the fusion image. Moreover, this edge enhancement is achieved via an actually physical effect instead of any image processing method. With additional post processing, it is predictable that even better images could be obtained.

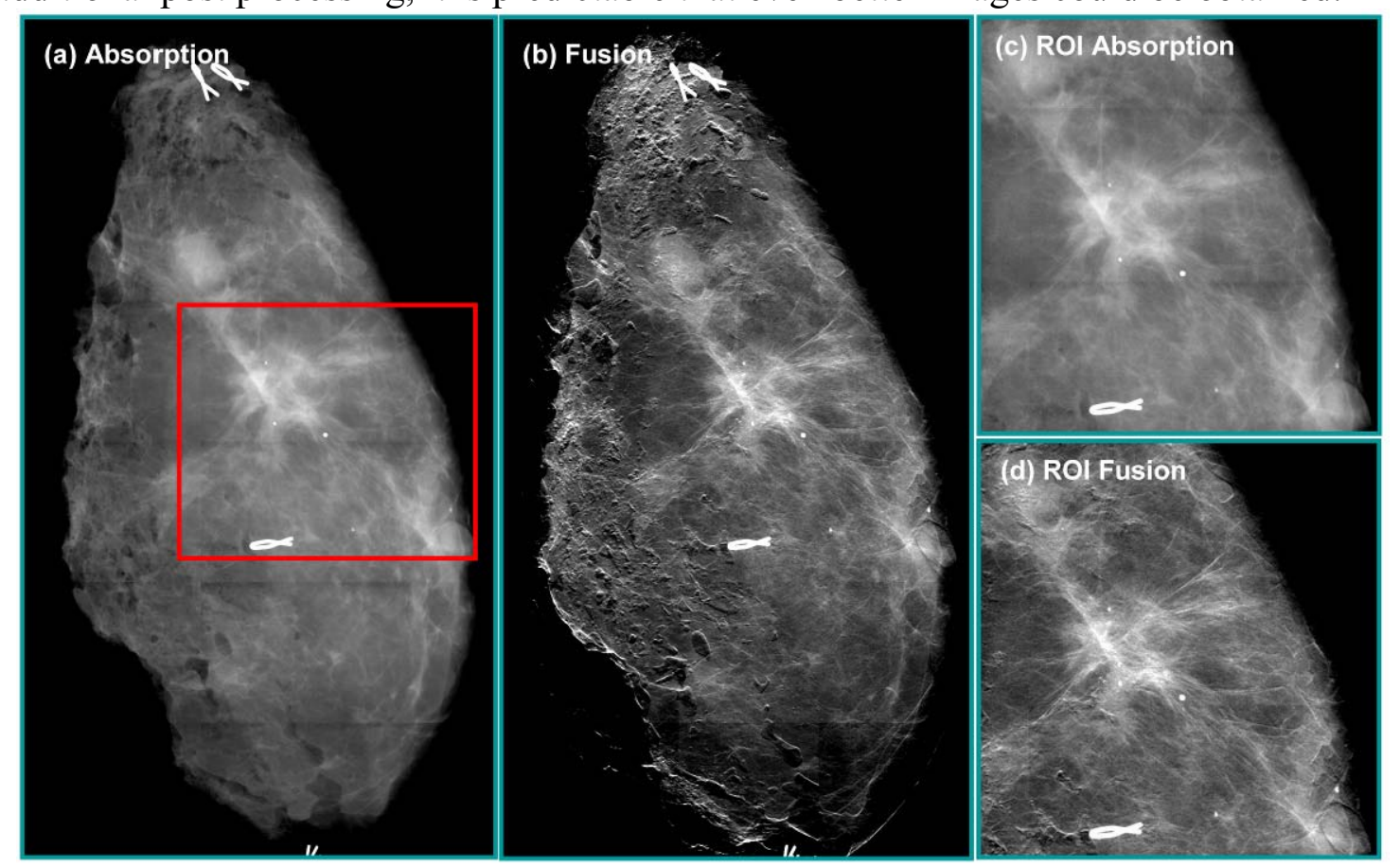

FIGURE 1. Comparison of the absorption-based mammography and PCA fusion of the MammoDPC. All the images are shown in the same display window. (a) The absorption image of a whole breast. (b) PCA fusion of the three components obtained by MammoDPC. (c) and (d) are the ROIs (the red rectangular in (a)) of the absorption image and fusion image, respectively.

Combining the preliminary outcomes from our first clinical study [5], we show that phase contrast and scattering imaging by the grating interferometer with an X-ray tubebased configuration and the dedicated image fusion algorithm can provide enhanced breast imaging to improve breast cancer diagnostics. Our technique is able to contribute to improved tumor and fibrous tissue visualization and to better discrimination between 
scars, inconspicuous breast tissue and invasive tumors [5]. Absorption and phase contrast enhanced images presented in this paper have been taken with the same dose. In particular for the breast specimens, the measured mean glandular dose (MGD) for a 4.5$\mathrm{cm}$ thick breast tissue was $26.2 \mathrm{mSv}$. This (high) dose is mainly due to a sub-optimal system design. It can be reduced to acceptable clinical values by a more careful design of several components, as discussed in Ref. [5].

\section{CONCLUSION}

In summary, native breast tissue has been investigated with an emerging differential phase contrast imaging technique based on a conventional X-ray source. Preliminary results confirm that this new approach can provide improved diagnostic contents with enhanced breast imaging.

\section{ACKNOWLEDGMENTS}

The authors thank G. Mikuljan of the Swiss Light Source (PSI) for designing and manufacturing the dedicated breast holder. They also thank N. Wieberneit and I. SchulzWenck of Philips Healthcare, Hamburg, Germany, for sharing their expertise in the interpretation of some MammoDPC images and the elucidation of their clinical value.

\section{REFERENCES}

1. R. A. Lewis, Phys. Med. Biol. 49, 3573 (2004).

2. C. David, B. Nöhammer, H. H. Solak, and E. Ziegler, Appl. Phys. Lett. 81, 3287-3289 (2002).

3. F. Pfeiffer, T. Weitkamp, O. Bunk, et al., Nature Physics 2(4), 258-261 (2006).

4. F. Pfeiffer, M. Bech, O. Bunk, et al., Nature Materials 7(2), 134-137 (2008).

5. M. Stampanoni, Z. Wang, T. Thuering, et al., Investigative Radiology 46 (12), 801 (2011). 